\title{
Fluoroscopic Guided Change of a Clogged Feeding Jejunostomy Tube in Superior Mesenteric Artery Syndrome
}

\author{
Preethi Jeyabal ${ }^{1}$, Kar Yin Seto ${ }^{2}$, Yuen Soon ${ }^{1}$ \\ Departments of ${ }^{1}$ General Surgery and ${ }^{2}$ Radiodiagnosis, Ng Teng Fong General Hospital, Singapore.
}

\section{Corresponding Author: \\ Dr. Yuen Soon}

Email:Yuen_soon@nuhs.edu.sg

This is an Open Access article distributed under the terms of the Creative Commons Attribution License (creativecommons.org/ licenses/by/3.0).

May 25, 2018

\begin{abstract}
Background: Blocked feeding jejunostomy tube is a common problem for which medical attention is sought. The problem of feeding jejunostomy tube clogging is common due to errors in medication regimen and feeds given through tube. Case Report: We describe a 63 year old with superior mesenteric artery syndrome, whose laparoscopically created feeding jejunostomy tube was clogged due to accidental feeding of resonium through the tube. Subsequently, the patient underwent a novel method of fluoroscopic guided replacement of jejunostomy tube, carried out without local anesthesia or sedation. Conclusion: The best way to deal with tube unclogging is to prevent it. Clear hospital protocols regarding the proper care of feeding tubes including medication should be available and reinforced to the nurses caring for patients on jejunostomy tube.
\end{abstract}

Keywords: Fluoroscopy, Gastrointestinal Intubation, Jejunostomy, Radiography, Parental Nutrition.

\section{Introduction}

The jejunostomy tube is an important means of access for enteral feeding in the surgical and medical patient. These tubes can be inserted surgically, endoscopically, radiologically, or laparoscopically [1]. A common complication of this method of feeding is tube occlusion. The rate of tube exchange due to clogging for radiologic and laparoscopic J-tubes was 2.3 and 0.9 per 1000 patient days respectively [2]. Although tube dislodgment/clogging is one of the most frequent mechanical problems associated with jejunostomy, there is a paucity of literature regarding the best techniques for catheter replacement.

We describe a patient with superior mesenteric artery (SMA) syndrome who's laparoscopically inserted jejunostomy tube was clogged because of administration of resonium through the tube. He successfully underwent fluoroscopic guided replacement of the clogged jejunostomy tube.

\section{Case Report}

A 63-year-old-male was admitted to the intensive care unit (ICU) for aspiration pneumonia following seizures, with background of previous subarachnoid hemorrhage due to a intracranial aneurysm. During the stay in ICU, he developed abdominal pain, distension and nasogastric output was high. Subsequent investigations lead to the diagnosis of SMA syndrome causing duodenal obstruction. Given the patient's general conditions and functional capacity, decision was made to insert feeding jejunostomy laparoscopically to address his nutritional requirements and hypoalbuminemia instead of a definitive surgery. Feeding jejunostomy was done laparoscopically at $15 \mathrm{~cm}$ from the duodeno-jejuanal junction using Witzel's technique.

Jejunostomy was functioning well as expected. But 5 days after creation of the jejunostomy tube, resonium was fed through the jejunostomy tube for the treatment of hyperkalemia. 
Resonium, being a highly viscous and sticky material, caused clogging of the jejunostomy tube. Multiple attempts to flush the tube and unclog the tube with wire were unsuccessful. Hence decision was made to change the feeding jejunostomy tube under fluoroscopic guidance.

A 9Fr sheath was placed over the feeding tube and inserted coaxially into the skin opening until it passes the jejunostomy. The blocked feeding tube was removed under fluoroscopy and replaced with a new feeding tube through the $9 \mathrm{Fr}$ sheath while injecting dilute contrast. The sheath was then removed after satisfactory placement of the feeding tube. Final position of the feeding tube was confirmed to be satisfactory without leakage and anchored to skin. Following replacement, jejunostomy tube was functioning well without issues [Fig.1].

\section{Discussion}

Superior mesenteric artery syndrome is a rare cause of high small bowel obstruction, caused by compression of the transverse part of the duodenum in between the superior mesenteric artery and aorta. Patients usually present with chronic abdominal pain, vomiting and weight loss. Jejunostomy tube can be clogged by number of factors including administration of medications that were not dissolved or crushed sufficiently, inadequate flushing of the tube, interactions of incompatible medications administered at the same time, deterioration of tube or balloon, burst balloon that is blocking the tube and kinking of tube [3]. Replacement under fluoroscopic guidance is considered safe and simple, and it is the preferred method in several centers; however, surgery remains the last option when endoscopic and radiologic methods are not successful [4].

Combining a clear plan for medication delivery with flushing will limit clogged tubes. Medication lists should be given a thorough review by a pharmacist to promote the use of available liquid alternatives; to limit the use of capsules

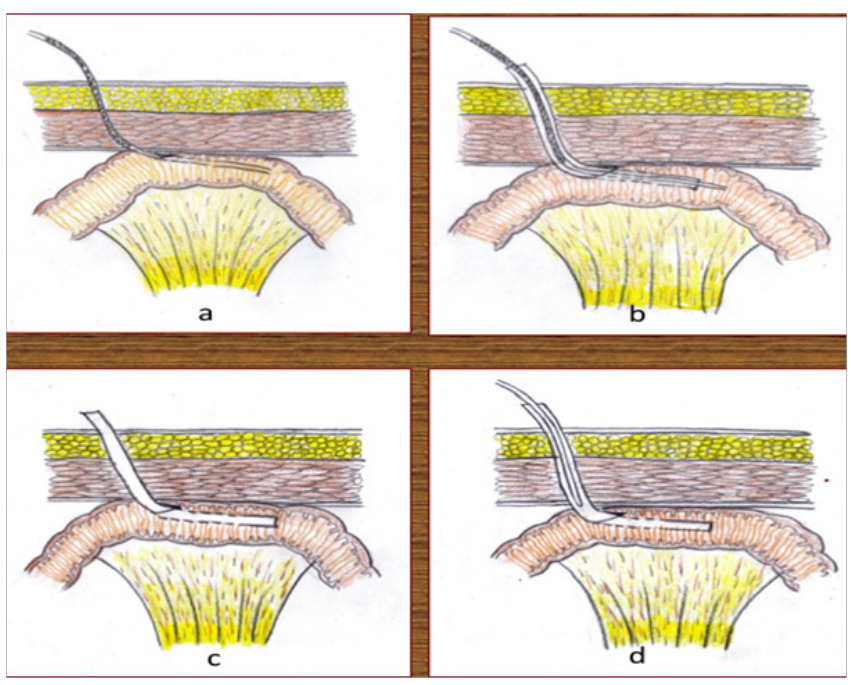

Fig.1(a): Blocked feeding jejunostomy tube due to resonium feeding. (b): $9 \mathrm{Fr}$ sheath placed over the jejunostomy tube, (c): the blocked tube removed under fuoroscopic guidance and (d): replaced with a new feeding jejunostomy tube through the 9 Fr sheath under fluoroscopic guidance while injecting dilute contrast.

and extended release forms; and to identify which pills can be finely crushed using a mortar and pestle (or other crushing device) and dissolved [6]. Pharmacists might also suggest alternative delivery modes such as intravenous, subcutaneous, intramuscular, transdermal, or rectal in order to limit the number of different medications that must be flushed down the feeding tube.

Several factors should be considered before administering medications through jejunostomy tube concomitantly with enteral nutrition (EN). Feeding tube size and placement site are among should be considered. Liquid preparations are the preferred formulations when possible because they are readily absorbed and are less likely to cause tube occlusions. Elixirs or suspensions are generally favored over syrups since syrups are more likely to cause clumping when exposed to EN [5-7]. Hypertonic medications, especially when undiluted, should not be administered directly into the small intestine, such as with a jejunostomy tube. Suspensions, which are often used for antibiotics, may be a more desirable type of liquid formulation. They generally contain less sorbitol than other 
liquid products, and most reconstituted antibiotic preparations have no sorbitol. Although suspensions may have a high osmolality, diluting with water will help decrease the tonicity [8]. Some liquid formulations are not appropriate for administration via the enteral tube. These include lansoprazole oral suspension granules and mineral oil, which are too viscous and may occlude the tube. Sucralfate suspension is also not suitable because it may cause an insoluble mass or bezoar formation [5-7]. Syrups should also be avoided, particularly when mixed with enteral formulas. Syrups with an acidic $\mathrm{pH}(\leq 4)$ are the most problematic because of their physical incompatibility with EN formulations, which can result in clumping or thickening and may cause the feeding tube to become clogged. Similar problems with tube occlusions may occur with ferrous sulfate elixir, which is not a syrup but is also very acidic. Some liquid preparations that are not compatible with enteral tube feedings and cause clumping or precipitation are listed in Table 1. Further diluting the syrup with water cannot prevent the physical incompatibilities between EN and syrups. An alternative liquid preparation is preferable. If this is not possible, the enteral feeding should be interrupted and the tube flushed with at least $30 \mathrm{~mL}$ of water before and after administering the incompatible syrup in order to separate the medication from the EN; the tube feeding may then resume [8]. Not all syrups are considered physically incompatible with enteral products. Syrups are generally less preferable to other liquid formulations because many syrups have $\mathrm{pH}$ values of $<4$ and cause compatibility problems.

Table 1: Partial list of medications to be avoided with jejonostomy tube.

\begin{tabular}{|c|c|c|}
\hline $\begin{array}{l}\text { Liquid medications with osmolality } \\
\text { of } \geq 3000 \mathrm{mosm} / \mathrm{kg}\end{array}$ & $\begin{array}{l}\text { Liquid medications } \\
\text { sorbitol }{ }^{[10,11]}\end{array}$ & $\begin{array}{l}\text { Liquid medications } \\
\text { enteral nutrition }{ }^{[9]}\end{array}$ \\
\hline 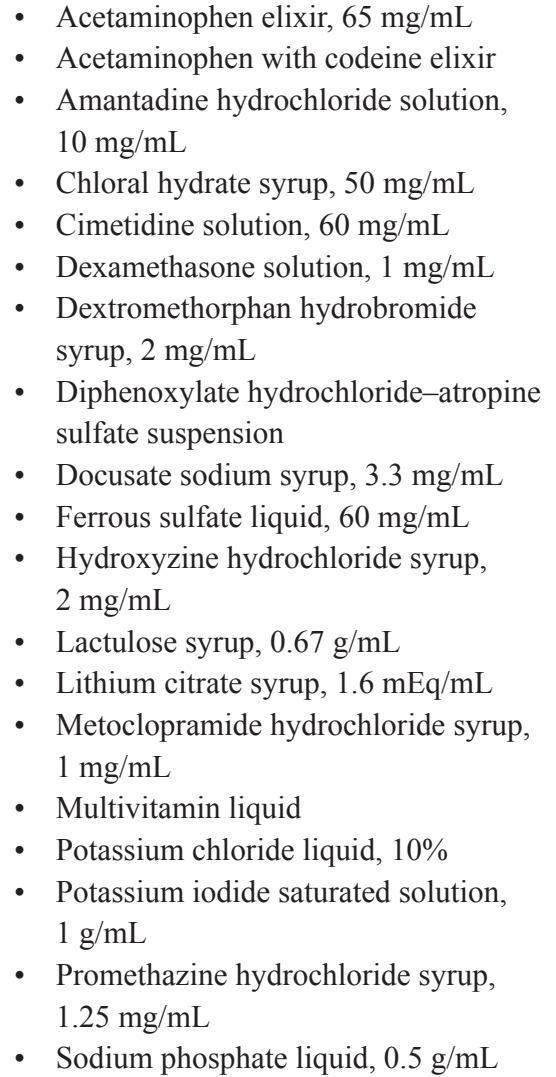 & $\begin{array}{l}\text { - Acetaminophen liquid } \\
\text { - Amantadine hydrochloride solution } \\
\text { - Aminocaproic acid syrup } \\
\text { - Charcoal liquid, with sorbitol } \\
\text { - Cimetidine solution } \\
\text { - Guaifenesin/dextromethorphan syrup } \\
\text { - Isoniazid syrup } \\
\text { - Lithium citrate syrup } \\
\text { - Metoclopramide hydrochloride syrup } \\
\text { - Phenylephrine hydrochloride/ } \\
\text { - brompheniramine maleate elixir } \\
\text { - Phenylephrine hydrochloride/ } \\
\text { - Phlorpheniramine maleate elixir } \\
\text { - Pseudoephedrine syrup } \\
\text { - Sodium polystyrene sulfonate } \\
\text { - } \text { suspension } \\
\text { - Tetracycline hydrochloride suspension }\end{array}$ & $\begin{array}{l}\text { Brompheniramine (Dimetane elixir, } \\
\text { Wyeth, Madison, NJ) } \\
\text { Calcium glubionate (Rugby, West } \\
\text { Hempstead, NY); } \mathrm{pH}=4 \\
\text { - Ferrous sulfate (Feosol elixir, } \\
\text { GlaxoSmith-Kline, London, England); } \\
\mathrm{pH}=2.2 \\
\text { - Guaifenesin (Robitussin liquid, Wyeth, } \\
\text { Madison, NJ) } \\
\text { - Lithium citrate (Cibalith-S syrup, CIBA } \\
\text { Pharmaceuticals, Summit, NJ); } \\
\text { pH =4.7-4.8 } \\
\text { Monobasic sodium phosphate (Fleet } \\
\text { Phospho-Soda, C.B. Fleet Co. Inc., Des } \\
\text { Moines, IA) } \\
\text { Potassium chloride liquid (Wyeth, } \\
\text { Madison, NJ) } \\
\text { Pseudoephedrine hydrochloride } \\
\text { (Sudafed syrup, Pfizer Inc., Brooklyn, } \\
\text { NY). }\end{array}$ \\
\hline
\end{tabular}


Tablets should be crushed to a fine powder using a mortar and pestle and then mixed with $15-30 \mathrm{~mL}$ of water before delivery through the tube. Similarly, hard gelatin capsules that contain a powdered drug may be opened and the contents diluted in 10-15 mL of water to form a slurry before administration [6-8]. Liquid filled soft gelatin capsule can be opened and contents squeezed out and administered. Alternately, the entire capsule can be dissolved in warm water and administered after filtering the undissolved soft gelatin particle.

Extended release capsules that contain some extended release granules may be opened and emptied into the feeding tube, but their contents should not be crushed. Examples of medications that may be administered this way include diltiazem and verapamil. Administer enteral medications via the oral route when possible. Determine the enteral feeding tube size (e.g., small bore or large bore), insertion site (e.g., nasal or percutaneous), tip location (e.g., stomach or jejunum), and enteral nutrition delivery method (e.g., continuous or bolus) $[5,9]$.

- Liquid dosage formulations are preferred.

- Elixirs and suspensions are generally favored over syrups.

- Check the sorbitol content if gastrointestinal distress occurs.

- Dilute hypertonic medications with at least 10$30 \mathrm{~mL}$ of water.

- If a solid dosage form is used, make sure the tablets can be crushed or the capsules opened.

- Feeding tubes should be flushed with 15-30 mL of water before and after drug delivery.

- When several medications are being given at the same time, each one should be administered separately. The feeding tube should be flushed with at least $5-10 \mathrm{~mL}$ of water between medications.

- Medications should not be directly mixed with the enteral feeding formulas.
- Watch for drug-nutrient interactions with continuous enteral feeding.

- Consider holding tube feeding for at least one to two hours before and after medication administration if drug absorption may be affected.

Clogged feeding tubes increases health care costs and decrease nutrient delivery. Consistent and scheduled flushing of all types of feeding tubes is the best defense against clogs. However, while avoiding clogged feeding tubes is a worthy goal, they can still occur. When they do, the front line methods for declogging are to use lukewarm water or carbonated beverage and failing which commercial products are available. Importantly, there is a gap in health care provider knowledge when it comes to best practices for declogging feeding tubes, and addressing this barrier will help prevent lost nutrition for patients, and it can be cost saving. With adequate knowledge and precautions, clogging of feeding jejunostomy tubes will become a rare occurrence and if it happens, fluoroscopic guided tube replacement is a simple and effective option to consider.

\section{Conclusion}

In patients receiving long-term enteral tube feeding, tube-related complications and problems are common and result in significant health care issue. The best way to deal with tube unclogging is to prevent it. Clear hospital protocols regarding the proper care of feeding tubes including medication that can be given should be available and reinforced to the nurses caring for patients on jejunostomy tube. Fluoroscopic guided jejunostomy tube replacement is feasible and safe in patients in whom traditional techniques of tube unclogging fail.

Contributors: PJ: manuscript writing, patient management; KYS: manuscript editing, imaging; YS: critical inputs into the manuscript and patient management. YS will act as guarantor. All authors approved the final version of this manuscript.

Funding: None; Competing interests: None stated. 


\section{References}

1. Vanek VW. Ins and Outs of Enteral Access. Part 3: LongTerm Access Jejunostomy. Nutr Clin Pract. 2003;18:5074.

2. Kim CY, Engstrom BI, Horvath J, Suhocki P, Smith TP Percutaneous primary jejunostomy tubes inserted using fluoroscopic guidance: comparison to laparoscopically inserted jejunostomy tubes. J Vasc Interv Radiol. 2013;24:S57- S58

3. Collares FB, Faintuch S, Kim SK, Rabkin DJ. Reinsertion of accidentally dislodged catheters through the original track: what is the likelihood of success? J Vasc Interv Radiol. 2010;21:861-864.

4. Tapia J, Murguia R, Garcia G, de los Monteros PE, Oñate E. Jejunostomy: techniques, indications, and complications. World J Surg. 1999;23:596-602.

5. Magnuson BL, Clifford TM, Hoskins LA, Bernard AC. Enteral nutrition and drug administration, interactions, and complications. Nutr Clin Pract. 2005;20:618-624.

6. Thomson FC, Naysmith MR, Lindsay A. Managing drug therapy in patients receiving enteral and parenteral nutrition. Hosp Pharmacist. 2000;7:155-164.

7. Dickerson RN, Melnik G. Osmolality of oral drug solutions and suspensions. Am J Hosp Pharm. 1988;45:832-834.

8. Nyffeler MS, Frankel E, Hayes E et al. Drug-nutrient interactions. In: Merritt R, DeLegge MH, Holcombe $\mathrm{B}$ et al., eds. The ASPEN nutrition support practice manual. 2nd ed. Silver Spring, MD: American Society for Parenteral and Enteral Nutrition; 2005:118-136.

9. Dickerson RN. Medication administration considerations for patients receiving enteral tube feedings. Hosp Pharm. 2004;39:84-89,96.

10. Lutomski DM, Gora ML, Wright SM, Martin JE. Sorbitol content of selected oral liquids. Ann Pharmacother. 1993;27:269-274.

11. Miller SJ, Oliver AD. Sorbitol content of selected sugarfree liquid medications. Hosp Pharm. 1993;28:741744,755 .

12. Myers JG, Page CP, Stewart RM, Schwesinger WH, Sirinek KR, Aust JB. Complications of needle catheter jejunostomy in 2022 consecutive applications. Am J Surg. 1995; 170:550-551. 